\title{
An Equilibrium Model for Commodity Prices with Regime Switching Reserve Dynamics
}

\author{
Karl Larsson* \\ Department of Economics \\ Knut Wicksell Centre for Financial Studies \\ School of Economics and Management \\ Lund University \\ FIRST VERSION: January 9, 2013 \\ THIS VERSION: March 11, 2014
}

\begin{abstract}
We explore the implications for asset prices and implied volatilities in an equilibrium model of commodity production. Production of the commodity can be carried out in one of two regimes. In the first regime the reserves are set in constant decline while in the second regime new additions to the reserve base are made. The optimal production rule is to switch regime when the stochastic revenue process of the producer hits certain barrier values. As a consequence of the optimal production rule equilibrium spot prices also become regime switching. The shapes of forward curves and implied volatilities are strongly dependent on the level of the stochastic revenue process.
\end{abstract}

JEL classification : Q30 Q40 G11 G13 M11 C61 C41 C44

Key words : Equilibrium, Commodity, Optimal switching, Impulse control, Forward curve

* Send correspondence to Karl Larsson, Department of Economics, Lund University, P.O Box 7082, S-220 07 Lund, Sweden. E-mail: karl.larsson@nek.lu.se, Phone: +46 (0)46 2228685 . Financial support from The Bank of Sweden Tercentenary Foundation is gratefully acknowledged. 


\section{Introduction}

It is widely recognized that prices of storable commodities such as oil, gas and minerals, are influenced by two central factors: reserves and inventories. In this paper we focus on the dynamic evolution of reserves over time and explore the implications for commodity prices in an infinite horizon model of industry equilibrium where production takes place in either of two regimes. Producers can either extract the resource from an existing reserve base or they can choose to make additions to the reserve base. These additions may e.g. be the result of intensified exploration leading to new discoveries of reserves, or they can be the result of the introduction of a new technology that allows for known but non-operating reserves to become profitable. Switching between these modes of operation the reserve base will either be in a state of decline or growth. There are costs associated with altering the mode of production that in general makes none of the regimes superior to the other. The producers optimal decision of when to change the method of production is solved in semi-closed form and depends on the uncertainties surrounding demand and the production process itself. The analysis is focused on the impact of reserve dynamics on spot prices and financial asset prices such as forward and standard option prices.

The resulting spot price model is a regime switching two-factor model consisting of the spot price $(S)$ and the stochastic part of revenues $(Z)$ as the two driving variables. The revenue variable $Z$ determines when it is optimal to switch regime. Regime switches occur when $Z$ crosses either a lower or an upper barrier. The variable $Z$ is stochastic and time varying and therefore gives rise to stochastic variation in both volatilities and the slope of the forward curve. We demonstrate that the variable $Z$ has a strong influence on both the slope of the forward curve and on the level of implied volatilities.

Equilibrium models of commodity prices has traditionally focused on storage as the main feature, or friction, impacting on equilibrium price formation, see e.g. Deaton and Laroque (1996), Routledge et al. (2000) and Evans and Guthrie (2010) for recent studies. A critique of storage models is that the inventory dynamics tend to have only short term effects and having very little impact on the long term properties of price levels, see e.g. Kogan et al. (2008).

Recent work has emphasized the production side of the economy together with frictions such as investment constraints and adjustment costs, see e.g. Kogan et al. (2008), Casassus et al. (2011), and Carlson et al. (2007). Kogan et al. (2008) study a model where the output commodity is produced from capital and where firms investments are irreversible and capacity constrained. The equilibrium spot price in their model is given by a one factor regime switching process. Our model is similar to theirs but we take the reserve base as the productive factor and allow firms explicitly to only operate in either of two regimes. Carlson et al. (2007) takes 
the reserve base as the sole input into production and couples it with adjustment costs. In their model reserves are exhaustible and the horizon is determined by the switch to a substitute technology. The main friction that drives their results however are non-linear adjustment costs. The model in Carlson et al. (2007) model is quite complex and all equilibrium quantities most be solved for numerically including the optimal production rule and spot prices. The model we propose yield an explicit production rule that only requires a single numerical input that is easily and quickly obtained. Similar to the models in Kogan et al. (2008) and Carlson et al. (2007) our model yields time varying volatilities and can also generate non-linear forward curves of different shapes. We also mention the study by Casassus et al. (2011) who studies a general equilibrium production economy where the consumption good is produced from two productive factors, capital and reserves. Their model features a non-linear Cobb-Douglas production function and irreversible investment. Also in their model a regime switching commodity price obtains as a result of the particular investment constraints they impose. Time varying volatilities also arises in equilibrium and are linked to the behavior of a single state variable. The same effect arises in our model although the mechanisms are different. In our model the level of price volatility and the slope of the forward curve are determined from a stochastic process, that is different from the spot price, and its distance to certain barrier values that triggers the switch to a different method of production. Similar to Kogan et al. (2008) and Carlson et al. (2007) we assume risk neutrality and hence cannot provide implications for risk premia. This is different from the general equilibrium setup in Casassus et al. (2011) who also obtain implications for the risk premium.

Financial asset prices based on commodities such as forward, futures and options are often modeled using reduced form models that are not linked to economic fundamentals. Both the storage and the production related equilibrium models typically result in a regime switching behavior of equilibrium prices. The regimes are related to decisions of either building or releasing inventory, or production and investment activities that either take place or not. The literature on reduced form models for storable commodity prices however has not included regime switching to a large extent. ${ }^{1}$ There is a large literature on reduced form models that can be used for application such as risk management and derivative pricing. A popular class of models are the Gaussian affine models. In these models, which may include multiple factors or state variables, the log spot price is an affine function of the state variables and in addition follows a normal (Gaussian) distribution. These models are highly tractable, see e.g. examples in Schwartz

\footnotetext{
${ }^{1}$ We remark that this discussion concerns the literature on storable commodities. In the literature on models for electricity prices the situation is different and regime switching has been included in many studies. The market for electrical power however is very different in many aspects from the markets for storable commodities and not covered by our model.
} 
(1997), Schwartz and Smith (2000), Miltersen and Schwartz (1998) and Casassus and CollinDufresne (2005). In recent years it has been recognized that to improve the realism and empirical performance of reduced form models it is necessary to include stochastic volatility and possibly also jumps. Schwartz and Trolle (2009) incorporate stochastic volatility in a multi factor model for a storable commodity, Casassus and Collin-Dufresene (2005) include jumps as do Hilliard and Reis (1998). Empirical evidence of stochastic volatility in the crude oil market can be found in Schwartz and Trolle (2009) and Larsson and Nossman (2011). Sørensen and Richter (2002) study soybean futures using a stochastic volatility model and Gudbrand and Koekkebakker (2004) find evidence of jumps in a study based on wheat futures prices. The regime switching models also induce stochastic volatility but the mechanism and resulting price processes can be quite different from the diffusive stochastic volatility specification typically employed in reduced form models. Vo (2009) presents empirical evidence of regime switching stochastic volatility in crude oil prices. Most reduced form models are of the so called affine, or exponentially affine, type. In exponentially affine models the log price is an affine function of possibly several state variables. These models are often highly tractable and therefore popular choices for applications such as derivative pricing. The equilibrium however tend not to give rise to affine spot prices. The models of e.g. Evans and Guthrie (2010), Kogan et al. (2008), Carlson et al. (2007) and Casassus et al. (2011) do not result in an affine structure for spot prices. In Kogan et al. (2008) the equilibrium spot price is however affine within the different regimes. The same is true for the storage model in Evans and Guthrie (2010). Casassus et al. (2011) approximates the equilibrium spot price in their model with an affine process within regimes. Also our model yield a regime switching spot price model that is affine within the different regimes.

The paper is organized as follows. In Section 1 we present the model and the resulting equilibrium production rule. In Section 2 we first present a numerical scenario that we then use to study asset prices numerically via Monte Carlo simulations. Section 3 concludes. Proofs and detailed calculations can be found in Appendices A and B.

\section{Industry equilibrium}

We model a continuous time infinite horizon economy. The economy consists of two types of agents; consumers and producers. For simplicity we assume that the supply side of the economy is made up of one monopolist producer. A model of a competitive industry organized by a social planner would result in similar conclusions. ${ }^{2}$ We assume that reserves constitute the only

\footnotetext{
${ }^{2}$ This point is discussed in more detail in Remark 1.
} 
productive factor and ignore capital and other inputs to production. ${ }^{3}$ Output at time $t$ is given by

$$
Q_{t}=q R_{t}
$$

where $q R_{t}$ denotes the amount of the extracted commodity with $q>0$ being the extraction rate and $R_{t}$ the current reserve size.

The evolution of reserves is governed by two different regimes depending on the firms current mode of operation. In the first regime, labelled $k=1$, reserves evolve according to the stochastic differential equation (SDE)

$$
d R_{1, t}=-q R_{1, t} d t+\sigma_{1} R_{1, t} d W_{R, t}
$$

where $W_{R, t}$ is a standard Brownian motion and $\sigma_{1}>0$. In this regime the firm makes no additions to its current supply of reserves. The total reserve therefore deteriorates at the fixed extraction rate $q$. In the second regime, $k=2$, the firm is able to add new reserves according to the rate $a>q$. The reserve dynamics in this regime are given by

$$
d R_{2, t}=(a-q) R_{2, t} d t+\sigma_{2} R_{2, t} d W_{R, t},
$$

where $\sigma_{2}>0$. In this regime the firm still extracts, and sells, the amount $q R_{t}$ at each instant but at the same time new reserves are added at the rate $a>0$. We allow for the volatility of the production process to be different in this regime, i.e. that $\sigma_{2} \neq \sigma_{1}$. This may be motivated from the fact that adding new reserves is a more complex operation than producing from a given reserve base which in turn may lead to a higher volatility. Defining

$$
b_{1}=-q \quad \text { and } \quad b_{2}=a-q
$$

we will write the dynamics of $R$ as

$$
d R_{t}=R_{t}\left(b_{\pi_{t}} d t+\sigma_{\pi_{t}} d W_{R, t}\right)
$$

where $\pi_{t}$ denotes an indicator of regimes and can only take on the values 1 or 2 . Note that the extraction rate $q$ is fixed and cannot be altered by the producer. However, the fact that the reserve base can be increased by switching to regime $k=2$ can be seen as a form of partial flexibility.

We abstract from running costs but assume that switching between the two regimes is costly for the firm. We denote the cost of switching from regime 2 to regime 1 by $\theta_{2,1}$ while the cost of

\footnotetext{
${ }^{3}$ It is relatively straightforward to introduce capital as a productive factor using a Cobb-Douglas production function in our setting. However, to keep the analysis focused on the impact of reserve dynamics we abstract from other productive factors.
} 
switching from regime 1 to regime 2 is denoted $\theta_{1,2}$. We will also make the following assumption about the switching costs:

$$
\theta_{1,2}>0 \quad \text { and } \quad \theta_{2,1}<0 .
$$

Moving from regime 1 to regime 2 is costly since adding new reserves will entail higher costs and therefore $\theta_{1,2}>0$. Moving from regime 2 to regime 1 on the other hand allows the firm to save on costs and potentially sell off equipment and capital that is no longer needed in the process of creating additions to the reserve base. Therefore we assume that the cost $\theta_{2,1}$ is negative.

Consumers are represented by an inverse demand curve given by

$$
S_{t}=Y_{t}^{\gamma} Q_{t}^{-\gamma}
$$

where $S$ is the spot price of the commodity and $Y$ represents demand shocks. We assume that $Y$ is given by the Geometric Brownian motion (GBM)

$$
d Y_{t}=\mu_{Y} Y_{t} d t+\sigma_{Y} Y_{t} d W_{Y, t}
$$

with $\mu_{Y} \in \mathbb{R}$ and $\sigma_{Y}>0$. If we define

$$
\xi(q)=q^{1-\gamma}
$$

then we can write the spot price as

$$
S_{t}=\xi(q) Y_{t}^{\gamma} R_{t}^{-\gamma}
$$

The instantaneous profit at $t$ from selling the quantity $Q$ at price $S$ can be written

$$
S_{t} Q_{t}=\xi(q) Y_{t}^{\gamma} R_{t}^{1-\gamma} \equiv \xi(q) Z_{t}
$$

where we in the last step have introduced the new state variable

$$
Z \equiv Y^{\gamma} R^{1-\gamma}
$$

which will have an important role in equilibrium.

We assume that production is carried out by a risk neutral monopolist firm which maximizes the discounted expected stream of profits.

Remark 1 We could also have assumed the existence of a social planner implementing a competitive equilibrium. Assuming the social planner is risk neutral and optimizes expected discounted consumer surplus the equilibrium would have the same properties as the one under monopoly. Define the surplus function as

$$
\int_{0}^{Q_{t}} S(x) d x=Y_{t}^{\gamma} \int_{0}^{Q_{t}} x^{-\gamma} d x
$$


where we view $S$ as a function of production $S=S(Q)$. Using (1) and assuming $\gamma<1$ we have

$$
\int_{0}^{Q_{t}} Y_{t}^{\gamma} x^{-\gamma} d x=\frac{q^{1-\gamma}}{1-\gamma} Y_{t}^{\gamma} R_{t}^{1-\gamma}
$$

such that the expected discounted consumer surplus becomes

$$
\mathbb{E}\left[\int_{0}^{\infty} e^{-r t}\left(\int_{0}^{Q_{t}} Y_{t}^{\gamma} x^{-\gamma} d x\right) d t\right]=\mathbb{E}\left[\int_{0}^{\infty} e^{-r t} \frac{\xi(q)}{1-\gamma} Z_{t} d t\right]
$$

which is the same quantity facing a monopolist with the only exception that $\xi(q)$ is now scaled by the factor $(1-\gamma)^{-1}>0$. This approach is used in e.g. Carlson et al. (2007), Kogan et al. (2008) and Evans and Guthrie (2010).

The firms optimization problem consists in choosing an optimal impulse control $\zeta=\left(\tau_{k}, \nu_{k}\right)$ consisting of a sequence of optimal switching times $\tau_{k}$, with $\tau_{k}<\tau_{k+1}$ and $\tau_{k} \rightarrow \infty$ a.s., and a sequence $\nu_{k}$ that takes values in $\{1,2\}$ and records the new regime entered into at time $\tau_{k}$. The set of all such impulse controls is denoted by $\mathcal{K}$. We define the total profit when the initial regime is $\pi_{0}=k$ and the firm uses an impulse control $\zeta \in \mathcal{K}$, as

$$
J_{k}(\zeta)=\mathbb{E}\left[\int_{0}^{\infty} e^{-r t} \xi(q) Z_{t} d t\right]-\mathbb{E}\left[\sum_{k=0}^{\infty} e^{-r \tau_{k}} \theta_{\nu_{k-1}, \nu_{k}}\right], \quad k=1,2
$$

where $r>0$ denotes the risk free interest rate which is assumed constant and satisfying the constraint $r>b_{2}=a-q$. The associated value function is obtained from choosing an optimal impulse control $\zeta$ that maximizes (11). The value functions corresponding to the total profits defined in (11) are

$$
V_{k}=\sup _{\zeta \in \mathcal{K}} J_{k}(\zeta), \quad k=1,2 .
$$

In Appendix A we give a more detailed account of the firms problem and how to solve it. Since our focus is on the dynamics of the equilibrium spot price and its implication for financial asset prices we only summarize the equilibrium results and state the optimal strategy. The functional forms for the value function, in the different regimes, are also presented in Appendix A.

A useful benchmark for the discussion of the equilibrium quantities are the cases when it is only possible to produce in either regime 1 or 2 with no switching allowed. It is straightforward to calculate the value functions under these assumptions and they are given by

$$
V_{k}^{\infty}=\mathbb{E}\left[\int_{0}^{\infty} e^{-r t} \xi(q) Z_{t} d t\right]=B_{k} Z_{0}, \quad k=1,2,
$$

where we have defined

$$
B_{k}=\frac{\xi(q)}{r-\delta_{1, k}}, \quad k=1,2
$$


and

$$
\delta_{1, k}=b_{k}(1-\gamma)+\mu_{Y} \gamma+\frac{1}{2} \gamma(\gamma-1)\left[\sigma_{Y}^{2}+\sigma_{k}^{2}\right], \quad k=1,2 .
$$

We will always impose the conditions

$$
r-\delta_{1, k}>0, \quad k=1,2,
$$

such that $V_{k}^{\infty}>0$ in both regimes $k=1,2$. To fix ideas we will assume that $B_{2}>B_{1}$ which implies that $V_{2}^{\infty}>V_{1}^{\infty}$ and hence that without switching regime 2 is preferred over regime 1 . Given this assumption the equilibrium production policy can be summarized as follows; when in regime 1 it is optimal to switch to regime 2 when the state variable $Z$ hits a barrier $K_{12}$, and when in regime 2 it is optimal to switch to regime 1 when $Z$ hits a lower threshold $K_{21}$ where $0<K_{21}<K_{12}$.

Before proceeding we need the following definitions

$$
\begin{aligned}
& \lambda_{1}^{+}=\frac{1}{2}\left(1-\frac{\delta_{1,1}}{\delta_{2,1}}\right)+\sqrt{\frac{1}{4}\left(1-\frac{\delta_{1,1}}{\delta_{2,1}}\right)^{2}+\frac{r}{\delta_{2,1}}} \\
& \lambda_{2}^{-}=\frac{1}{2}\left(1-\frac{\delta_{1,2}}{\delta_{2,2}}\right)-\sqrt{\frac{1}{4}\left(1-\frac{\delta_{1,2}}{\delta_{2,2}}\right)^{2}+\frac{r}{\delta_{2,2}}},
\end{aligned}
$$

where

$$
\delta_{2, k}=\frac{1}{2}\left[\sigma_{Y}^{2} \gamma^{2}+\sigma_{k}^{2}(1-\gamma)^{2}\right]
$$

and where $\delta_{1, k}$ are given by (15) for $k=1,2$. Then the barriers $K_{12}$ and $K_{21}$ are explicitly given by

$$
\begin{aligned}
& K_{12}=\frac{\theta_{1,2} \lambda_{1}^{+} \lambda_{2}^{-}\left(\omega^{\lambda_{2}^{-}}-\omega^{\lambda_{1}^{+}}\right)}{B_{2}-B_{1}}\left[\lambda_{1}^{+} \lambda_{2}^{-}\left(\omega^{\lambda_{2}^{-}}-\omega^{\lambda_{1}^{+}}\right)+\lambda_{1}^{+}\left(\omega^{\lambda_{1}^{+}}-\omega\right)+\lambda_{2}^{-}\left(\omega-\omega^{\lambda_{2}^{-}}\right)\right]^{-1}, \\
& K_{21}=\omega K_{12} .
\end{aligned}
$$

where $\omega$ solves the equation

$$
\frac{h_{1}(\omega)}{h_{2}(\omega)}-\frac{\theta_{1,2}}{\theta_{2,1}}=0
$$

with the functions $h_{1}$ and $h_{2}$ defined by

$$
\begin{aligned}
& h_{1}(\omega)=\lambda_{1}^{+} \lambda_{2}^{-}\left(\omega^{1+\lambda_{1}^{+}}-\omega^{1+\lambda_{2}^{-}}\right)+\lambda_{1}^{+}\left(\omega^{1+\lambda_{2}^{-}}-\omega^{\lambda_{1}^{+}+\lambda_{2}^{-}}\right)+\lambda_{2}^{-}\left(\omega^{\lambda_{1}^{+}+\lambda_{2}^{-}}-\omega^{1+\lambda_{1}^{+}}\right) \\
& h_{2}(\omega)=\lambda_{1}^{+} \lambda_{2}^{-}\left(\omega^{\lambda_{2}^{-}}-\omega^{\lambda_{1}^{+}}\right)+\lambda_{1}^{+}\left(\omega^{\lambda_{1}^{+}}-\omega\right)+\lambda_{2}^{-}\left(\omega-\omega^{\lambda_{2}^{-}}\right) .
\end{aligned}
$$

The value of $\omega$ must be solved for numerically from (20) but this can be done very quickly using standard methods. 
Regime switches are governed by the process $Z$ and thus it is worthwhile studying its properties more closely. Note that the dynamics of the process $Z$ when it is in regime $k$ can, by application of Itô's formula, be written as

$$
d Z_{k, t}=Z_{k, t}\left(\mu_{Z, k} d t+\sigma_{Z, k} d W_{Z, t}\right)
$$

where $W_{Z, t}$ is a standard Brownian motion and

$$
\mu_{Z, k}=\gamma \mu_{Y}+(1-\gamma) b_{k}+\frac{1}{2} \gamma(\gamma-1)\left(\sigma_{k}^{2}+\sigma_{Y}^{2}\right) \quad ; \quad \sigma_{Z, k}=\sqrt{\gamma^{2} \sigma_{Y}^{2}+(1-\gamma)^{2} \sigma_{k}^{2}}
$$

for $\mathrm{k}=1,2$. While it is not possible to study most of the properties of this model analytically it is possible to calculate the probabilities

$$
H_{12}(t)=P\left(\tau_{12}<t \mid Z_{0}<K_{12}, \pi_{0}=1\right)
$$

and

$$
H_{21}(t)=P\left(\tau_{21}<t \mid Z_{0}>K_{21}, \pi_{0}=2\right)
$$

where

$$
\tau_{12}=\inf _{t \geq 0}\left\{Z_{t}=K_{12} \mid Z_{0}<K_{12}, \pi_{0}=1\right\}
$$

and

$$
\tau_{21}=\inf _{t \geq 0}\left\{Z_{t}=K_{21} \mid Z_{0}<K_{21}, \pi_{0}=2\right\}
$$

are the first times the upper and lower barriers are hit by the process $Z$ respectively conditional upon starting at $Z_{0}$ in either regime $\pi_{0}=1$ or $\pi_{0}=2$. The cumulative distribution functions $H_{12}$ and $H_{21}$ are given by

$$
H_{12}(t)=N\left(\frac{-\alpha_{12}+\beta_{1} t}{\sigma_{Z, 1} \sqrt{t}}\right)+e^{2 \alpha_{12} \beta_{1}} N\left(\frac{-\alpha_{12}-\beta_{1} t}{\sigma_{Z, 1} \sqrt{t}}\right)
$$

and

$$
H_{21}(t)=N\left(\frac{\alpha_{21}-\beta_{2} t}{\sigma_{Z, 2} \sqrt{t}}\right)+e^{2 \alpha_{21} \beta_{2}} N\left(\frac{\alpha_{21}-\beta_{2} t}{\sigma_{Z, 2} \sqrt{t}}\right)
$$

where

$$
\alpha_{k n}=\log \left(Z_{k n} / Z_{0}\right) \quad \text { and } \quad \beta_{k}=\mu_{Z, k}-\frac{1}{2} \sigma_{Z, k}^{2}, \quad k=1,2, \quad n=1,2, \quad k \neq n .
$$

We refer to Appendix B for proofs of these formulas. The distribution functions $H_{k n}(t)$ thus gives us the probability that, conditional on the process $Z$ being in regime $k$, will switch to regime $n$ before time $t$. 


\section{$3 \quad$ Equilibrium asset prices}

We now turn to the implications of the equilibrium for the formation of spot, forward and option prices. We first present some theoretical results and then give some numerical examples. Since neither forward or options prices can be derived in closed form in equilibrium we study aspects of these prices numerically using Monte Carlo simulations.

\subsection{Spot prices}

The spot price is by the definition (7) determined by two stochastic factors; reserves $(R)$ and demand shocks $(Y)$. Furthermore it will be a regime switching process. Applying Itô's formula to $S=(Q / Y)^{\gamma}=(q R / Y)^{\gamma}$ and using the dynamics (3) and (8) we find that the SDE of the spot price in regime $k$ is given by

$$
d S_{k, t}=S_{k, t}\left(\mu_{S, k} d t+\sigma_{k} d W_{R, t}+\sigma_{Y} d W_{Y, t}\right),
$$

where

$$
\mu_{S, k}=\gamma\left(\mu_{Y}-b_{k}\right)+\frac{1}{2} \gamma(1+\gamma) \sigma_{k}^{2}+\frac{1}{2} \gamma(\gamma-1) \sigma_{Y}^{2}
$$

and

$$
\sigma_{S, k}=\sqrt{\gamma^{2} \sigma_{k}^{2}+\gamma^{2} \sigma_{Y}^{2}}
$$

for $k=1,2$. Spot prices of commodities are often stated in terms of the cost of carry which is determined by the relevant interest rate and the convenience yield for the commodity. In our setting the interest rate is constant and equal to $r$. The drift of the spot price $\mu_{S, k}$ must equal the cost of carry which is given by $r-\varrho_{k}$ where $\varrho_{k}$ denotes the convenience yield in regime $k$. It follows that the convenience yield is recovered from $\varrho_{k}=r-\mu_{S, k}$.

\section{$3.2 \quad$ Forward prices}

The forward price at $t$ and with maturity $T$ is in this economy given by

$$
F_{k}(t, T)=\mathbb{E}_{t}\left[S_{T}\right], \quad k=1,2
$$

where the subscript $k$ indicates the initial regime that governs the production process $R$ at time $t$. Note that in our setting with deterministic interest rates and no credit risk forward and futures prices will coincide. There exist no closed form solution for the forward price in (29) and we therefore study them using Monte Carlo simulation.

Before proceeding we note that if there were no switching between production processes then the forward price would be

$$
F_{k}^{\infty}(t, T)=\mathbb{E}_{t}\left[S_{k, T}\right]=S_{0} e^{\mu_{k, S}(T-t)}, \quad k=1,2 .
$$


The forward prices in (30) are useful as benchmarks since one may expect that if the state variable $Z$ is far above the switching barrier $K_{12}$ (and hence in regime 2), the likelihood of crossing $K_{21}$ (and hence moving into regime 1) is comparably low at least for short to medium long maturities. Therefore at least the short end of the forward curve should resemble the curve $F_{2}^{\infty}(t, T)$. A similar argument applies to the case when $Z$ have attained low values below $K_{21}$ (and hence is in regime 1) and the probability of switching into regime 2 in the near future is low. In this case the short end of the forward curve should resemble $F_{1}^{\infty}(t, T)$. When $Z$ is located between the barriers we expect the forward curve to lie between these two curves.

\subsection{Option prices}

We also study option prices and in particular the implied volatilities obtained from these. The price at time $t$ of a European call option written on the spot price $S$ and with maturity at time $T$ is in this economy given by

$$
C(t, T, K)=e^{-r(T-t)} \mathbb{E}_{t}\left[\left(S_{T}-K\right)^{+}\right]
$$

where $x^{+}=\max (x, 0)$ for $x \in \mathbb{R}$. There is no closed form expression for this price and we calculate it via Monte Carlo simulation.

We define the implied volatility as the input volatility $\bar{\sigma}$ that solves the equation

$$
C(t, T, K)-C^{B}(t, T, K ; \bar{\sigma})=0
$$

where $C^{B}(t, T, K ; \sigma)$ is given by Blacks formula

$$
C^{B}(t, T, K ; \sigma)=e^{-r(T-t)}\left(F(t, T) N\left(d_{1}\right)-K N\left(d_{2}\right)\right)
$$

with $F(t, T)=\mathbb{E}_{t}\left[S_{T}\right]$, and

$$
d_{1}=\frac{\ln (F(t, T) / K)+\frac{1}{2} \sigma^{2}(T-t)}{\sigma \sqrt{T-t}} \quad ; \quad d_{2}=\frac{\ln (F(t, T) / K)-\frac{1}{2} \sigma^{2}(T-t)}{\sigma \sqrt{T-t}}
$$

and with $N(x)$ denoting the cumulative distribution function of a standard normal random variable with mean zero and unit variance. Note that in order to compute $C^{B}(t, T, K ; \sigma)$ we need a Monte Carlo estimate of $F(t, T)$ since it is not available on closed form.

As in the case with forward curves it is possible to calculate benchmark option prices assuming there is no switching. If the only available regime is either $k=1$ or $k=2$ then the price of the European call option is given by

$$
C_{k}^{\infty}\left(t, T, K, \sigma_{S, k}\right)=e^{-r(T-t)}\left(F_{k}^{\infty}(t, T) N\left(d_{1, k}\right)-K N\left(d_{2, k}\right)\right)
$$


where $F_{k}^{\infty}$ are given by (30), and

$$
d_{1, k}=\frac{\ln \left(F_{k}^{\infty}(t, T) / K\right)+\frac{1}{2} \sigma_{S, k}^{2}(T-t)}{\sigma_{S, k} \sqrt{T-t}} \quad ; \quad d_{2, k}=\frac{\ln \left(F_{k}^{\infty}(t, T) / K\right)-\frac{1}{2} \sigma_{S, k}^{2}(T-t)}{\sigma_{S, k} \sqrt{T-t}}
$$

with $\sigma_{S, k}$ defined in (28) for $k=1,2$. Note that $\sigma_{S, k}$ is the implied volatility in each regime $k$. The corresponding prices, $P^{B}(t, T, K ; \sigma)$ and $P_{k}^{\infty}\left(t, T, K, \sigma_{S, k}\right)$, for European put options can also easily be calculated but we refrain from stating these expressions.

\section{$4 \quad$ Numerical examples}

In this section we study pricing of forward contracts and spot options numerically. We start by discussing a set of benchmark parameters that we will used through most of the following examples. In table 1 we have presented two sets of parameters and also included some calculated equilibrium quantities such as the switching barriers and switching probabilities. We will base most of our examples on the first set of parameters (Set I) and below we will mainly discuss the implications of this choice of parameters. The second parameter set (set II) in table 1 will only be used for numerical comparisons of asset prices and we therefore do not discuss it in detail. The main difference between the two sets are that the set II features higher volatilities than set I.

\section{INSERT TABLE 1 AROUND HERE}

First we set the parameter $q$ that determines the rate of production in both regimes to $q=0.02$ and hence $b_{1}=-q=-0.02$. This means that if production remained in regime 1 for ever (such that there are no additions to reserves) then the expected reserve size $\mathbb{E}\left[R_{t}\right]$ will equal 10 percent of the initial reserve (i.e. $\mathbb{E}\left[R_{t}\right]=R_{0} / 10$ ) after $t \approx 115$ years. Alternatively the expected value of the first time $\tau$ that the reserve hits the value $R_{0} / 10$ is $\mathbb{E}[\tau] \approx 92$ years. ${ }^{4}$ Note that this analysis only applies to the situation when the possibility to add reserves is ignored and therefore only serves to establish some intuition for the value of $q$. We assume that when the process is in regime 2 reserves are added at the rate $a=0.04$ such that the net additions occurs at the rate $b_{2}=a-q=0.02$. Further, we also assume that the production process is more volatile in the regime 2 when reserves are added which is a more complex operation than

\footnotetext{
${ }^{4}$ Note that if the production process remains in regime 1 forever the expected value of the reserve size is $\mathbb{E}\left[R_{t}\right]=R_{0} \exp (-q t)$. Solving for $t$ from the condition $\mathbb{E}\left[R_{t}\right]=R_{0} / N$ yields $t=\ln (N) / q$. A more precise way of addressing the time it takes for the production process $R$ to attain a particular fraction $(1 / N)$ of the initial reserve $R_{0}$ is to calculate the expected value of the stopping time $\tau=\inf \left\{t \mid R_{t} \leq R_{0} / N\right\}$. Such results are available and indeed the expected value is explicitly given by the expression $\mathbb{E}[\tau]=\ln (N) /\left(q+0.5 \sigma_{1}^{2}\right)$, see e.g. $\varnothing \mathrm{ksendal}$ (2002).
} 
producing from and existing reserve base as it is done in regime 1 . We therefore set $\sigma_{1}=0.1$ and $\sigma_{2}=0.2$. The parameters of the demand shock are set to $\mu_{Y}=0.01$ and $\sigma_{Y}=0.3$. Thus the demand shock is expected to increase demand by $1 \%$ on a yearly basis while being quite volatile. Further we set $r=0.1$ and $\gamma=0.5$. Finally we set the switching costs to $\theta_{1,2}=8.5$ and $\theta_{2,1}=-8.4$. Recall that the switching costs can be thought of as a way of capturing the costs of being in each regime in reduced form. With this choice of parameters the value functions of being in either regime 1 or 2 forever are

$$
V_{1}^{\infty}\left(Z_{0}\right) \equiv \frac{\xi(q)}{r-\delta_{1,1}} Z_{0}=30.3158 \quad \text { and } \quad V_{2}^{\infty}\left(Z_{0}\right) \equiv \frac{\xi(q)}{r-\delta_{1,2}} Z_{0}=39.0367 .
$$

The switching costs thus constitutes the fractions $\theta_{1,2} / V_{1}^{\infty}=0.2804$ and $\theta_{2,1} / V_{2}^{\infty}=0.2152$, of the long term values in (34). The barriers for switching are $K_{12}=25.1879$ and $K_{21}=19.6202$. We typically set initial values according to $R_{0}=1$ and set $Y_{0}=Z_{0}^{1 / \gamma}$. Then the initial spot price is given by $S_{0}=q^{-\gamma} Y_{0}^{\gamma}=q^{-\gamma} Z_{0}$. As an example; if we set the initial value of the state variable $Z$ to be halfway between the barriers $K_{12}$ and $K_{21}$, i.e. $Z_{0}=\left(K_{12}+K_{21}\right) / 2$, then $Z_{0}=22.4040$ and $S_{0}=158.4203$. In table 1 we have also calculated the probabilities $P_{k n}(t)$ for $t=0.5$ and $t=1$ years for the baseline case where $Z_{0}=\left(K_{12}+K_{21}\right) / 2$. These probabilities indicate that for this values of $Z_{0}$ regime 2 is more short-lived than regime 1 . We also plotted these probabilities in figure 1 as functions of different time horizons $t$ and for three different values of $Z_{0}$. From figure 1 it is evident that the probabilities are highly dependent on how close $Z$ is to the switching barriers. In the left panel of figure 1 we have that $Z_{0}=0.5 \times\left(K_{12}+K_{21}\right)$ and in this case the probabilities $H_{12}(t)$ and $H_{21}(t)$ are very close for values of $t \leq 2$ years. For longer time horizons $t$ the probability of switching to regime 1 is substantially higher than the probability of switching to regime 1 . In the middle panel we have set $Z_{0}=0.995 \times K_{12}$ and naturally the probability $H_{12}$ is now much higher. The right panel show the opposite situation where $Z_{0}=1.05 \times K_{21}$ is very close to the barrier $K_{21}$ where it is optimal to switch from regime 2 to 1 .

\section{INSERT FIGURE 1 AROUND HERE}

We define contango as a situation where the forward curve is upward sloping, and backwardation as a situation where the forward curves is downward sloping. ${ }^{5}$ The choice of baseline parameters results in the following drift terms for the spot price dynamics in each regime

$$
\mu_{S, 1}=0.0075>0 \quad \text { and } \quad \mu_{S, 2}=-0.0112<0 .
$$

\footnotetext{
${ }^{5}$ These definitions can be refined, see e.g. Litzenberger and Rabinowitz (1995) who distinguish between weak and strong backwardation, but we believe that they are standard and sufficient for the purpose of illustrating the effect of regime switching on the qualitative shape of the forward curve.
} 
This means that absent any regime switching the regime 1 will feature a forward curve in contango and regime 2 will feature a forward curve in backwardation. The corresponding spot price volatilities in each regime become

$$
\sigma_{S, 1}=0.1581 \quad \text { and } \quad \sigma_{S, 2}=0.1802 .
$$

So, the spot price is in this case more volatile in regime 2 than in regime 1.

\subsubsection{Forward curves}

We study forward curves as functions of some key parameters. First we look at graphs that display forward curves for different initial values of $Z_{0}$.

In figure 2 we have set $Z_{0}=0.5 \times\left(K_{12}+K_{21}\right)$ and plotted the forward prices $F_{1}(0, T)$, $F_{2}(0, T), F_{1}^{\infty}(0, T)$ and $F_{2}^{\infty}(0, T)$ for maturities ranging from $T=0$ to $T=1$ years. Since the forward price always equals the spot price when $T=0$ all curves originate at the same point $S_{0}$. Furthermore, the curves $F_{1}^{\infty}(0, T)$ and $F_{2}^{\infty}(0, T)$ serve as upper and lower bounds for the equilibrium forward curves $F_{1}(0, T)$ and $F_{2}(0, T)$. When $Z_{0}=\left(K_{12}+K_{21}\right) / 2$ the state variable is exactly half way between the barriers where the firm switches its method of production. What we see is that the curve $F_{2}$ is in backwardation while flattening out at a higher level compared to that of the downward sloping limit curve $F_{2}^{\infty}$. The curve $F_{1}$ is in contango but also flattens for longer maturities. The short end of $F_{1}$ is sloping upwards, reflecting the fact that the system is operating in regime 1 and that a switch in the near future is unlikely. The long end of the curve starts to slope downwards for maturities above 0.7 years, reflecting an increased probability of a switch when looking at longer time horizons. These curves demonstrate the models ability to generate non-linear forward curves with different shapes in equilibrium.

\section{INSERT FIGURE 2 AROUND HERE}

Figure 3 shows the same curves when $Z_{0}=0.995 \times K_{12}$ and thus is very close to the upper barrier $K_{12}$. In this case we find that both the equilibrium forward curves are in backwardation. The curve $F_{2}$ is in this case quite close to the limit curve $F_{2}^{\infty}$. Note that also that the equilibrium curve $F_{1}$ when $Z$ is in regime 1 is also in strong backwardation. Hence, regime 1 cannot be labeled as a contango regime; the graph shows that the curve $F_{1}$ moves from contango to backwardation before the actual switch to regime 2 .

\section{INSERT FIGURE 3 AROUND HERE}

The situation is reversed in figure 4 where the state variable $Z$ is set to $Z_{0}=1.05 \times K_{21}$ and thus is close to the lower barrier $K_{21}$. In this case the curve $F_{1}$ is in strong contango and approaches 
its limit curve $F_{1}^{\infty}$. The curve $F_{2}$, while still in (weak) backwardation in the short end, tends to have an inverted hump-shape with the long end of the curve bending slightly upwards.

\section{INSERT FIGURE 4 AROUND HERE}

Quantitatively we observe e.g. in figure 2 that even tough the regimes are relatively long lived their presence impact on the forward curve already in what must be regarded as the short to medium part of the curve.

Parameter set II features more sharp contango and backwardation limits and higher levels of volatility in both regimes. In figure 5 we have plotted the same forward curves as in figure 2 but with the second set of parameters. The general picture is the same as that communicated by 2 .

\section{INSERT FIGURE 5 AROUND HERE}

\subsubsection{Implied volatilities}

We also study the at-the-money implied volatility as a function of the state variable $Z$. Recall that a standard European call or put option is said to be at-the-money when the strike price $K$ equals the initial spot price $K=S_{0}$. Regardless of what regime the production process is in the at-the-money volatility will be an increasing function of $Z$. This feature is shown in figure 6 where we have plotted the at-the-money implied volatility as a function of $Z$; there is one curve showing the implied volatility when the current regime is $k=1$ and when when the regime is $k=2$. In both cases the implied volatility is an increasing function of $Z$. This is because regardless of the current regime the location of the state variable $Z$ alters the probability of switching regime. When the current regime is $k=1$ a high value of $Z$ that is close to reaching the barrier $K_{12}$ is an indication that the process is about to switch to regime $k=2$ where the instantaneous volatility of the spot price is higher. When the current regime is $k=2$ and the process $Z$ is close to the barrier $K_{21}$ the likelihood of a switch to regime $k=1$ is comparably high.

\section{INSERT FIGURE 6 AROUND HERE}

In our examples implied volatility increases with $Z$ while the slope of the forward curve typically decreases. Hence, the model is able to generate the sometimes observed feature that backwardation tends to coincide with higher levels of volatility. In figure 7 we have plotted implied volatility as a function of $Z$ but now using the parameter set II in table 1 . This graph shows the same pattern as that in figure 6 .

\section{INSERT FIGURE 7 AROUND HERE}




\section{Concluding remarks}

We have explored the implications for spot and futures prices and implied volatilities in an equilibrium model of commodity production. Production of the commodity can only be carried out in one of two regimes; in the first regime the reserves are set in constant decline while in the second regime new additions to the reserve base are made and the total amount of reserves may be increasing. The optimal production rule is regime switching and the optimal times to switch the mode of production are determined by hitting times where the stochastic revenues $Z$ of the producer hits certain barrier values. As a consequence of the optimal production rule spot prices also become regime switching.

We demonstrate that the model is able to generate nonlinear forward curves. The shapes of the forward curves are strongly dependent on the level of the state variable $Z$ in particular on its distnace to the barrier levels at which the firm switches its production regime. Implied volatilities are also strongly dependent on the state variable $Z$ and can therefore display significant time variation.

Finally we emphasize that similar to most equilibrium models we have explored a model that is highly stylized. There are many features that may be relevant to commodity price formation that are absent from our formulation. We have focused on the single mechanism of reserve adjustments.

\section{References}

[1] Black, F., 1976. The pricing of commodity contracts. Journal of Financial Economics, 3, 167-179.

[2] Carlson, M., Khoker, Z., Titman, S., 2007. Equilibrium exhaustible resource price dynamics, Journal of Finance, Vol. 42, 1663-1703.

[3] Casassus, J., Collin-Dufresne, P., Routledge, B., 2011. Equilibrium commodity prices with irreversible investment and non-linear technology, SSRN Working paper series.

[4] Casassus, J., Collin-Dufresne, P., 2005. Stochastic convenience yield implied from commodity futures and interest rates. Journal of Finance, Vol. 60, No. 5, 2283-2331.

[5] Doran, J. S., Ronn, E. I., 2008. Computing the market price of volatility risk in the energy commodity markets, Journal of Banking and Finance, Vol. 32, 2541-2552.

[6] Deaton, A., Laroque, G., 1998. Competitive storage and commodity price dynamics, Journal of Political Economy, 104(5), 826-923. 
[7] Evans, L., Guthrie, G., 2010. Commodity prices and the option value of storage, SSRN working paper series.

[8] Geman, H., Shih, Y. F., 2009. Modeling commodity prices under the CEV model. Journal of Alternative Investments, Vol. 11, No. 3, 65-84.

[9] Gudbrand, L., Koekebakker, S., 2004. Volatility and price jumps in agricultural futures prices - evidence from wheat options. American Journal of Agricultural Economics, Vol. 86, No. 4, 1018-1031.

[10] Hilliard, J. E., Reis, J., 1998. Valuation of commodity futures and options under stochastic convenience yields, interest rates, and jump diffusiosn in the spot, Journal of Financial and Quantitative Finance, 33, 61-86.

[11] Karatzas, I., Shreve, S. E., 1996. Brownian Motion and Stochastic Calculus. Springer Verlag.

[12] Kogan, L., Livdan, D., Yaron, A., 2008. Oil futures prices in a production economy with investment constraints, Journal of Finance, Vol. 64, 1345-1375.

[13] Larsson, K., Nossman, M., 2011. Jumps and stochastic volatility in oil prices: time series evidence, Energy Economics, Vol. 33, No. 3, 504-514.

[14] Litzenberger, R. H., Rabinowitz, N., 1995. Backwardation in oil futures markets: theory and empirical evidence, Journal of Finance, Vol. 50, 1517-1545.

[15] Miltersen, K., Schwartz, E. S., 1998. Pricing of options on commodity futures with stochastic term structures of convenience yields and interest rates, Journal of Financial and Quantiative Analysis, 33, 33-59.

[16] Musiela, M., Rutkowski, M., 1998. Martingale Methods in Financial Modelling. Springer Verlag.

[17] Pham, H., Ly Vath, V., 2007. Explicit solution to an optimal switching problem in the two regime case, SIAM Journal on Control and Optimization, 395-426.

[18] Routledge, B., Seppi, D., Spatt, C., 2000. Equilibrium forward curves for commodities, Journal of Finance, Vol. 55, 1297-1338.

[19] Schwartz, E. S., 1997. The stochastic behavior of commodity prices: Implications for valuation and hedging. Journal of Finance, Vol. 52, No. 3, 923-973. 
[20] Schwartz, E. S., Smith, J. E., 2000. Short-term variations and long-term dynamics in commodity prices. Management Science, Vol. 47, No. 2, 893-911.

[21] Schwartz, E. S., Trolle, A., 2009. Unspanned stochastic volatility and the pricing of commodity derivatives. Review of Financial Studies, Vol. 22, 4423-4461.

[22] Sorensen, C., Richter, M. C., 2002. Stochastic volatility and seasonality in commodity futures and options: the case of soybeans. SSRN working paper series.

[23] Vo, M. T., 2009. Regime-switching stochastic volatility: evidence from the crude oil market. Energy Economics, 31, 779-788. 


\section{Appendix A}

In this Appendix we provide some details of the model results in section ??. For a detailed and rigorous presentation of the theory we refer to Pham and Ly Vath (2007).

Note that the value functions $V_{1}$ and $V_{2}$ are both functions of $R$ and $Y$ and we write $V_{k}=$ $V_{k}(R, Y)$ to emphasize this fact. Below we show that the value functions can be expressed in terms of the single state variable $Z=Y^{\gamma} R^{1-\gamma}$. First we define the Itô operators $\mathcal{L}_{k}$ that operates on a function $\phi=\phi(R, Y)$ via

$$
\mathcal{L}_{k} \phi=b_{k} R \frac{\partial \phi}{\partial R}+\mu_{Y} Y \frac{\partial \phi}{\partial Y}+\frac{1}{2} \sigma_{k}^{2} R^{2} \frac{\partial^{2} \phi}{\partial R^{2}}+\frac{1}{2} \sigma_{Y}^{2} Y^{2} \frac{\partial^{2} \phi}{\partial Y^{2}}, \quad k=1,2 .
$$

As described in Pham and Ly Vath (2007) the value functions $V_{1}$ and $V_{2}$ must satisfy the following system of variational inequalities

$$
\begin{aligned}
& \min \left\{r V_{1}-\mathcal{L}_{1} V_{1}-\Pi, V_{1}-\left(V_{2}-\theta_{1,2}\right)\right\}=0 \\
& \min \left\{r V_{2}-\mathcal{L}_{2} V_{2}-\Pi, V_{2}-\left(V_{1}-\theta_{2,1}\right)\right\}=0 .
\end{aligned}
$$

A basic requirement in order to solve the above system is that we can solve the partial differential equations

$$
r V_{k}(Y, R)-\mathcal{L}_{k} V_{k}(Y, R)-\Pi(Y, R)=0, \quad k=1,2 .
$$

where we recall that we have the profit flow $\Pi(Y, R)=Y^{\gamma} \xi(q) R^{1-\gamma}=\xi(q) Z$.

The value function that solves (A.4) depends on the state vector $(R, Y)$ but the homogeneity of the inverse demand function suggests that the number of state variables can be reduced. Indeed, the value function can be written on the form

$$
V_{k}(R, Y)=\phi_{k}(Z), \quad k=1,2 .
$$

for some functions $\phi_{k}$. The HJB equations (A.4) in each regime then reduces to the following ordinary differential equations for the function $\phi_{k}$ :

$$
\xi(q) Z-r \phi(Z)+\delta_{1, k} \phi^{\prime}(Z) Z+\delta_{2, k} \phi^{\prime \prime}(Z) Z^{2}=0, \quad k=1,2,
$$

where $\delta_{1, k}$ and $\delta_{1, k}$ are defined in (15) and (19), and $\delta_{1, k}$ satisfies the condition in (16).

The general solution to the equation in (A.6) is given by

$$
\phi_{k}(Z)=A_{1, k} Z^{\lambda_{k}^{-}}+A_{2, k} Z^{\lambda_{k}^{+}}+B_{k} Z
$$

for $k=1,2$. In (A.6) $A_{n, k}$ for $n=1,2$ and $k=1,2$ are constants to be determined,

$$
B_{k}=\frac{\xi(q)}{r-\delta_{1, k}}, \quad k=1,2,
$$


and $\lambda_{k}^{-}$and $\lambda_{k}^{+}$are the roots of the quadratic equation $h_{k}(\lambda)=0$ where

$$
h_{k}(\lambda)=-r+\delta_{1, k} \lambda+\delta_{2, k} \lambda(\lambda-1), \quad k=1,2 .
$$

The roots to the equations in (A.9) are explicitly given by

$$
\lambda_{k}^{ \pm}=\frac{1}{2}\left(1-\frac{\delta_{1, k}}{\delta_{2, k}}\right) \pm \sqrt{\frac{1}{4}\left(1-\frac{\delta_{1, k}}{\delta_{2, k}}\right)^{2}+\frac{r}{\delta_{2, k}}}
$$

Since $h_{k}(0)=-r<0$ and $h_{k}(1)=-\left(r-\delta_{1, k}\right)<0$, by (16), we have $\lambda_{k}^{-}<0$ and $\lambda_{k}^{+}>1$ in both regimes $k=1,2$. If there were no switching between regimes the value functions could be calculated as

$$
V_{k}^{\infty}(Z)=\mathbb{E}\left[\int_{0}^{\infty} e^{-r t} \xi(q) Y_{t}^{\gamma} R_{t}^{1-\gamma} d t\right]=B_{k} Z
$$

This result allows us to write the value functions as

$$
V_{k}(Z)=A_{1, k} Z^{\lambda_{k}^{+}}+A_{2, k} Z^{\lambda_{k}^{-}}+V_{k}^{\infty}(Z), \quad k=1,2 .
$$

From this representation we may interpret the terms $A_{1, k} Z^{\lambda_{k}^{+}}+A_{2, k} Z^{\lambda_{k}^{-}}$as the option values associated with the ability to switch to different modes of production. Assuming that $B_{2}>B_{1}$ then regime switches will occur at barrier points $0<K_{21}<K_{12}$. Hence, in regime 1 we switch to regime 2 when $Z$ hits the barrier $K_{12}$ from below, and when in regime 2 we switch to regime 1 when $K_{21}$ is hit from above. We may then impose the following natural economic conditions on the option values:

$$
\lim _{Z \rightarrow 0}\left(A_{1,1} Z^{\lambda_{1}^{+}}+A_{2,1} Z^{\lambda_{1}^{-}}\right)=0 \quad ; \quad \lim _{Z \rightarrow \infty}\left(A_{1,2} Z^{\lambda_{2}^{+}}+A_{2,2} Z^{\lambda_{2}^{-}}\right)=0 .
$$

These conditions states that the option value of being able to switch to regime 1 should tend to zero as $Z \rightarrow \infty$, and that the option value of being able to switch to regime 2 should tend to zero when $Z \rightarrow 0$. Since $\lambda_{1}^{+}>1$ and $\lambda_{2}^{-}<0$ the conditions in (A.13) imply that $A_{1,2}=A_{2,1}=0$. Relabeling the constants $A_{1,1}$ and $A_{2,2}$ according to $A_{2,2} \equiv A_{2}$ and $A_{1,1} \equiv A_{1}$ we can write the candidate value functions as

$$
V_{1}(Z)=A_{1} Z^{\lambda_{1}^{+}}+B_{1} Z \quad \text { and } \quad V_{2}(Z)=A_{2} Z^{\lambda_{2}^{-}}+B_{2} Z
$$

Pham and Ly Vath (2007) prove that the structure of the switching regions, and hence the optimal switching policy, is determined by the existence of two constant barriers $K_{12}$ and $K_{21}$. The optimal switching rule can then be expressed as follows: when the process $Z$ is in regime $k=1$ it is optimal to switch to regime $k=2$ when the process $Z$ hits the barrier $K_{12}$ and when the process $Z$ is in regime $k=2$ it is optimal to switch to regime $k=1$ when $Z$ hits the barrier 
$K_{21}$. Assume that $V_{2}^{\infty}>V_{1}^{\infty}$ so that without any switching the regime $k=2$ is preferred over regime $k=1$. Then we have $K_{12}>K_{21}>0$ and the value functions

$$
V_{1}(Z)=\left\{\begin{array}{cc}
A_{1} Z^{\lambda_{1}^{+}}+B_{1} Z, & Z<K_{12} \\
V_{2}(Z)-\theta_{1,2}, & Z \geq K_{12}
\end{array}\right.
$$

and

$$
V_{2}(Z)=\left\{\begin{array}{rl}
V_{1}(Z)-\theta_{2,1} & Z \leq K_{21} \\
A_{2} Z^{\lambda_{2}^{-}}+B_{2} Z, & Z>K_{21}
\end{array}\right.
$$

solves the required variational inequalities in (A.2) and (A.3).

What remains is to determine the constants $A_{1}, A_{2}, K_{12}$ and $K_{21}$. This is done from the boundary conditions

$$
V_{1}\left(K_{12}\right)+\theta_{1,2}=V_{2}\left(K_{12}\right) \quad ; \quad V_{1}\left(K_{21}\right)-\theta_{2,1}=V_{2}\left(K_{21}\right)
$$

and

$$
V_{1}^{\prime}\left(K_{12}\right)=V_{2}^{\prime}\left(K_{12}\right) \quad ; \quad V_{1}^{\prime}\left(K_{21}\right)=V_{2}^{\prime}\left(K_{21}\right) .
$$

These conditions states that the value functions match at the switching points and that they are continuously differentiable at these points. With the expressions for the value functions the conditions at the switching points translate into the following system of equations

$$
\begin{aligned}
A_{1} K_{12}^{\lambda_{1}^{+}}+B_{1} K_{12}+\theta_{1,2} & =A_{2} K_{12}^{\lambda_{2}^{-}}+B_{2} K_{12} \\
A_{1} K_{21}^{\lambda_{1}^{+}}+B_{1} K_{21}-\theta_{2,1} & =A_{2} K_{21}^{\lambda_{2}^{-}}+B_{2} K_{21} \\
\lambda_{1}^{+} A_{1} K_{12}^{\lambda_{1}^{+}-1}+B_{1} & =\lambda_{2}^{-} A_{2} K_{12}^{\lambda_{2}^{-}-1}+B_{2} \\
\lambda_{1}^{+} A_{1} K_{21}^{\lambda_{1}^{+}-1}+B_{1} & =\lambda_{2}^{-} A_{2} K_{21}^{\lambda_{2}^{-}-1}+B_{2} .
\end{aligned}
$$

To solve the system it is helpful to write it as

$$
\left[\begin{array}{cccc}
1 & 0 & K_{12}^{\lambda_{1}^{+}} & -K_{12}^{\lambda_{2}^{-}} \\
0 & 1 & K_{21}^{\lambda_{1}^{+}} & -K_{21}^{\lambda_{2}^{-}} \\
0 & 0 & \lambda_{1}^{+} K_{12}^{\lambda_{1}^{+}} & -\lambda_{2}^{-} K_{12}^{\lambda_{2}^{-}} \\
0 & 0 & \lambda_{1}^{+} K_{21}^{\lambda_{1}^{+}} & -\lambda_{2}^{-} K_{21}^{\lambda_{2}^{-}}
\end{array}\right]\left(\begin{array}{c}
\theta_{1,2} \\
-\theta_{2,1} \\
A_{1} \\
A_{2}
\end{array}\right)=\left(B_{2}-B_{1}\right)\left(\begin{array}{c}
K_{12} \\
K_{21} \\
K_{12} \\
K_{21}
\end{array}\right)
$$

This system can, with obvious definitions, be written $K x=y$. Solving the above system for $x$ such that $x=K^{-1} y$ one may deduce that the ratio $\theta_{1,2} /\left(-\theta_{2,1}\right)$ only depends on the variable $\omega \equiv K_{21} / K_{12}$. Furthermore the value of $\omega$ solves the equation

$$
\frac{h_{1}(\omega)}{h_{2}(\omega)}-\frac{\theta_{1,2}}{\theta_{2,1}}=0
$$


with the functions $h_{1}$ and $h_{2}$ given by

$$
\begin{aligned}
& h_{1}(\omega)=\lambda_{1}^{+} \lambda_{2}^{-}\left(\omega^{1+\lambda_{1}^{+}}-\omega^{1+\lambda_{2}^{-}}\right)+\lambda_{1}^{+}\left(\omega^{1+\lambda_{2}^{-}}-\omega^{\lambda_{1}^{+}+\lambda_{2}^{-}}\right)+\lambda_{2}^{-}\left(\omega^{\lambda_{1}^{+}+\lambda_{2}^{-}}-\omega^{1+\lambda_{1}^{+}}\right) \\
& h_{2}(\omega)=\lambda_{1}^{+} \lambda_{2}^{-}\left(\omega^{\lambda_{2}^{-}}-\omega_{1}^{\lambda_{1}^{+}}\right)+\lambda_{1}^{+}\left(\omega^{\lambda_{1}^{+}}-\omega\right)+\lambda_{2}^{-}\left(\omega-\omega^{\lambda_{2}^{-}}\right) .
\end{aligned}
$$

Having solved for $\omega$ from (A.17) the first two equations in (A.16) imply that the barriers may be expressed according to

$$
\begin{aligned}
K_{12} & =\frac{\theta_{1,2} \lambda_{1}^{+} \lambda_{2}^{-}\left(\omega^{\lambda_{2}^{-}}-\omega^{\lambda_{1}^{+}}\right)}{B_{2}-B_{1}}\left[\lambda_{1}^{+} \lambda_{2}^{-}\left(\omega^{\lambda_{2}^{-}}-\omega^{\lambda_{1}^{+}}\right)+\lambda_{1}^{+}\left(\omega^{\lambda_{1}^{+}}-\omega\right)+\lambda_{2}^{-}\left(\omega-\omega^{\lambda_{2}^{-}}\right)\right]^{-1}, \\
K_{21} & =\omega K_{12} .
\end{aligned}
$$

Finally, the following expressions for the constants $A_{1}$ and $A_{2}$ can easily be derived from the last two equations of the same system and we have

$$
\begin{aligned}
& A_{1}=\frac{B_{2}-B_{1}}{\lambda_{1}^{+}}\left(\frac{K_{21} K_{12}^{\lambda_{2}^{-}}-K_{12} K_{21}^{\lambda_{2}^{-}}}{K_{12}^{\lambda_{2}^{-}} K_{21}^{\lambda_{1}^{+}}-K_{12}^{\lambda_{1}^{+}} K_{21}^{\lambda_{2}^{-}}}\right) \\
& A_{2}=\frac{B_{2}-B_{1}}{\lambda_{2}^{-}}\left(\frac{K_{21} K_{12}^{\lambda_{1}^{+}}-K_{12} K_{21}^{\lambda_{1}^{+}}}{K_{12}^{\lambda_{2}^{-}} K_{21}^{\lambda_{1}^{+}}-K_{12}^{\lambda_{1}^{+}} K_{21}^{\lambda_{2}^{-}}}\right) .
\end{aligned}
$$

This completes the proof. Since we do not study the value functions we do note report the expressions for $V_{k}$ and $A_{k}$ in the text. 


\section{Appendix B}

In this Appendix we provide some details regarding the distribution functions $H_{12}(t)$ and $H_{21}(t)$ given in equations (23) and (24). First recall that we defined the stopping times

$$
\tau_{12}=\inf _{t \geq 0}\left\{Z_{t}=K_{12} \mid Z_{0}<K_{12}, \pi_{0}=1\right\}
$$

and

$$
\tau_{21}=\inf _{t \geq 0}\left\{Z_{t}=K_{21} \mid Z_{0}<K_{21}, \pi_{0}=2\right\} .
$$

The associated distribution functions are

$$
H_{12}(t)=\mathbb{P}\left(\tau_{12} \leq t\right) \quad \text { and } \quad H_{21}(t)=\mathbb{P}\left(\tau_{21} \leq t\right) .
$$

In regime $k$ the process $Z$ has the explicit solution

$$
Z_{k, t}=Z_{0} \exp \left\{\left(\mu_{Z, k}-\frac{1}{2} \sigma_{Z, k}^{2}\right) t+\sigma_{Z, k} W_{Z, t}\right\}
$$

where we used the equality in distribution $\sigma_{Z, k} W_{Z, t} \stackrel{d}{=} \gamma \sigma_{Y} W_{Y, t}+(1-\gamma) \sigma_{k} W_{R, t}$, with $W_{Z, t}$ denoting a standard Brownian motion.

The hitting time $\tau_{12}=\inf _{t \geq 0}\left\{Z_{1, t}=K_{12}\right\}$ can thus be expressed as

$$
\begin{aligned}
\tau_{12} & =\inf _{t \geq 0}\left\{\ln \left(Z_{1, t}\right)=\ln \left(K_{12}\right)\right\} \\
& =\inf _{t \geq 0}\left\{W_{Z, t}+\left(\frac{\mu_{Z, 1}-\frac{1}{2} \sigma_{Z, 1}^{2}}{\sigma_{Z, 1}}\right) t=\frac{\ln \left(K_{12} / Z_{0}\right)}{\sigma_{Z, 1}}\right\} \\
& =\inf _{t \geq 0}\left\{W_{Z, t}+\left(\frac{\beta_{1}}{\sigma_{Z, 1}}\right) t=\frac{\alpha_{12}}{\sigma_{Z, 1}}\right\}
\end{aligned}
$$

where we used the definitions of $\alpha_{12}$ and $\beta_{1}$ in (25).

Furthermore, since we condition on $\left\{K_{12}>Z_{0}\right\}$ we have that $\alpha_{12}=\ln \left(K_{12} / Z_{0}\right)>0$ and we may write

$$
\mathbb{P}\left(\tau_{12} \leq t\right)=\mathbb{P}\left(\max _{s \in[0, t]}\left\{W_{Z, t}+\left(\frac{\beta_{1}}{\sigma_{Z, 1}}\right) t\right\} \geq \frac{\alpha_{12}}{\sigma_{Z, 1}}\right)
$$

The last line of the above calculation show that we require the distribution of the maximum of a standard Brownian motion with drift. This is a well known result in stochastic analysis, see e.g. Corollary B.3.2, p.470 in Musiela and Rutkowski (1998). Using this result we find that the distribution function for $\tau_{12}$ is given by

$$
\mathbb{P}\left(\tau_{12} \leq t\right)=N\left(\frac{-\alpha_{12}+\beta_{1} t}{\sigma_{Z, 1} \sqrt{t}}\right)+e^{2 \alpha_{12} \beta_{1}} N\left(\frac{-\alpha_{12}-\beta_{1} t}{\sigma_{Z, 1} \sqrt{t}}\right) .
$$


For the probability $H_{21}$ we can use similar considerations and we first note that

$$
\tau_{21}=\inf _{t \geq 0}\left\{W_{Z, t}+\left(\frac{\beta_{2}}{\sigma_{Z, 2}}\right) t=\frac{\alpha_{21}}{\sigma_{Z, 2}}\right\} .
$$

In this case we have that $K_{21}<Z_{0}$ and hence that $\alpha_{21}=\ln \left(K_{21} / Z_{0}\right)<0$. Therefore we can write

$$
\mathbb{P}\left(\tau_{21} \leq t\right)=\mathbb{P}\left(\min _{s \in[0, t]}\left\{W_{Z, t}+\left(\frac{\beta_{2}}{\sigma_{Z, 2}}\right) t\right\} \leq \frac{\alpha_{21}}{\sigma_{Z, 2}}\right) .
$$

The distribution of the minimum of a standard Brownian motion is also a standard result, see e.g. Corollary B.3.4, p.470 in Musiela and Rutkowski (1998). Using this result the required distribution function for $\tau_{21}$ reads

$$
\mathbb{P}\left(\tau_{21} \leq t\right)=N\left(\frac{\alpha_{21}-\beta_{2} t}{\sigma_{Z, 2} \sqrt{t}}\right)+e^{2 \alpha_{21} \beta_{2}} N\left(\frac{\alpha_{21}+\beta_{2} t}{\sigma_{Z, 2} \sqrt{t}}\right) .
$$


Tables and Figures 

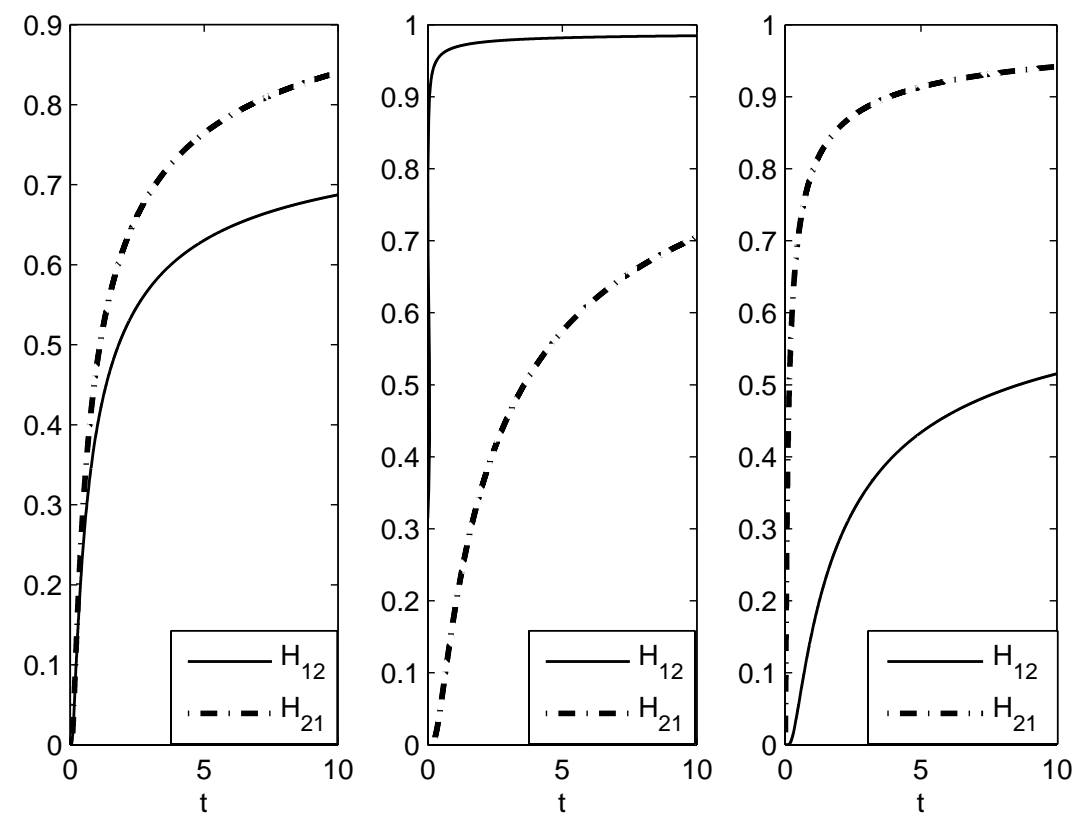

Figure 1: The Probabilities $H_{12}(t)$ and $H_{21}(t)$ as functions of $t$ for three different values of $Z_{0}$. The left panel has $Z_{0}=\left(K_{12}+K_{21}\right) / 2$, the middle panel has $Z_{0}=0.995 \times K_{12}$, and the right panel has $Z_{0}=1.05 \times K_{21}$. Parameters are set according to parameter set I. 


\begin{tabular}{|c|c|c|}
\hline & Set I & Set II \\
\hline \multicolumn{3}{|l|}{ Parameters } \\
\hline$r$ & 0.1 & 0.1 \\
\hline$q$ & 0.02 & 0.02 \\
\hline$a$ & 0.06 & 0.1 \\
\hline$\sigma_{1}$ & 0.1 & 0.2 \\
\hline$\sigma_{2}$ & 0.2 & 0.3 \\
\hline$\mu_{Y}$ & 0.01 & 0.01 \\
\hline$\sigma_{Y}$ & 0.3 & 0.4 \\
\hline$\gamma$ & 0.5 & 0.7 \\
\hline$b_{1}$ & -0.02 & -0.02 \\
\hline$b_{2}$ & 0.04 & 0.08 \\
\hline$\theta_{1,2}$ & 8.5 & 8.5 \\
\hline$\theta_{2,1}$ & -8.4 & -8.4 \\
\hline \multicolumn{3}{|c|}{ Equilibrium quantities } \\
\hline$\mu_{S, 1}$ & 0.0075 & 0.0280 \\
\hline$\mu_{S, 2}$ & -0.0112 & -0.0420 \\
\hline$\sigma_{S, 1}$ & 0.1581 & 0.3130 \\
\hline$\sigma_{S, 2}$ & 0.1803 & 0.3500 \\
\hline$K_{12}$ & 25.1879 & 13.7140 \\
\hline$K_{21}$ & 19.6202 & 9.4389 \\
\hline $\mathbb{P}\left(\tau_{12}<1\right)$ & 0.3956 & 0.4843 \\
\hline $\mathbb{P}\left(\tau_{21}<1\right)$ & 0.4759 & 0.5321 \\
\hline $\mathbb{P}\left(\tau_{12}<0.5\right)$ & 0.2549 & 0.3531 \\
\hline $\mathbb{P}\left(\tau_{21}<0.5\right)$ & 0.3071 & 0.3565 \\
\hline
\end{tabular}

Table 1: Parameters and calculated equilibrium quantities for scenarios I and II. 


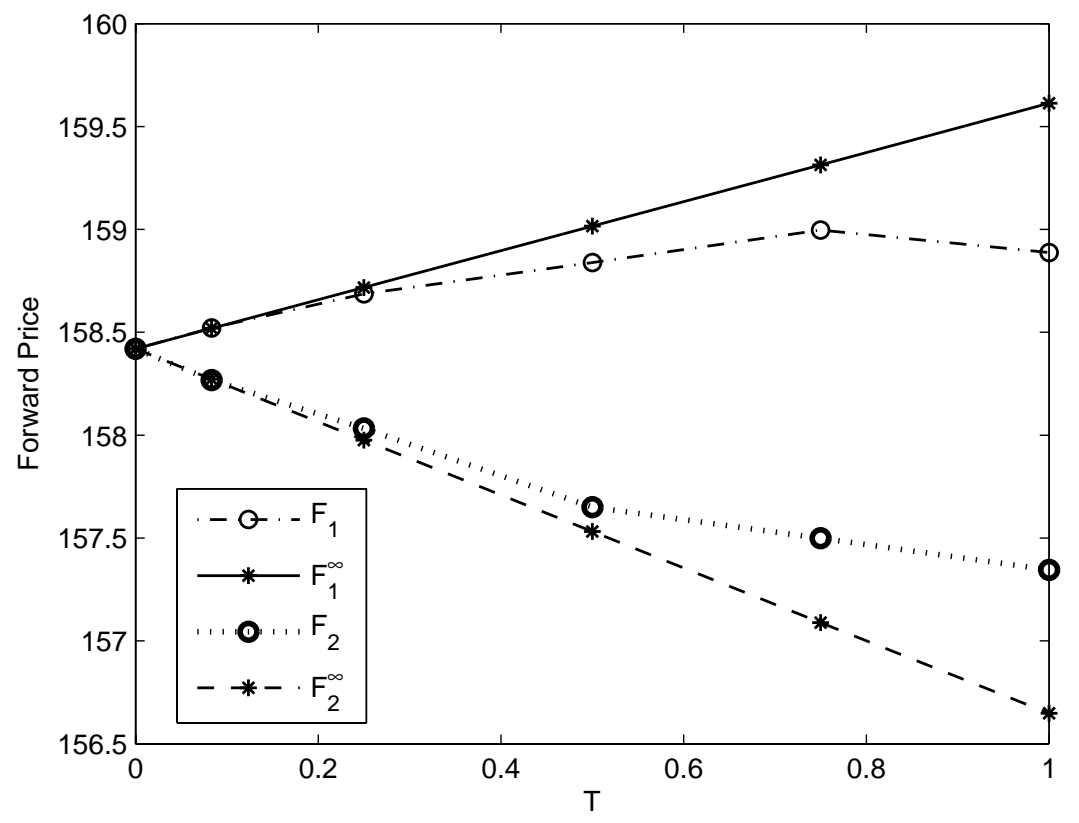

Figure 2: Forward prices, at $t=0$, plotted for time to maturities between $T=0$ and $T=1$ year. The initial value of the state variable is set to $Z_{0}=\left(K_{12}+K_{21}\right) / 2$ in all curves and the current spot price is $S_{0}$. The current regime for the curves $F_{1}$ and $F_{1}^{\infty}$ is $k=1$, and the current regime for the curves $F_{2}$ and $F_{2}^{\infty}$ is $k=2$. Each point on the curves $F_{1}$ and $F_{2}$ is calculated from 600,000 paths of the underlying spot price process. Parameters are set according to parameter set I. 


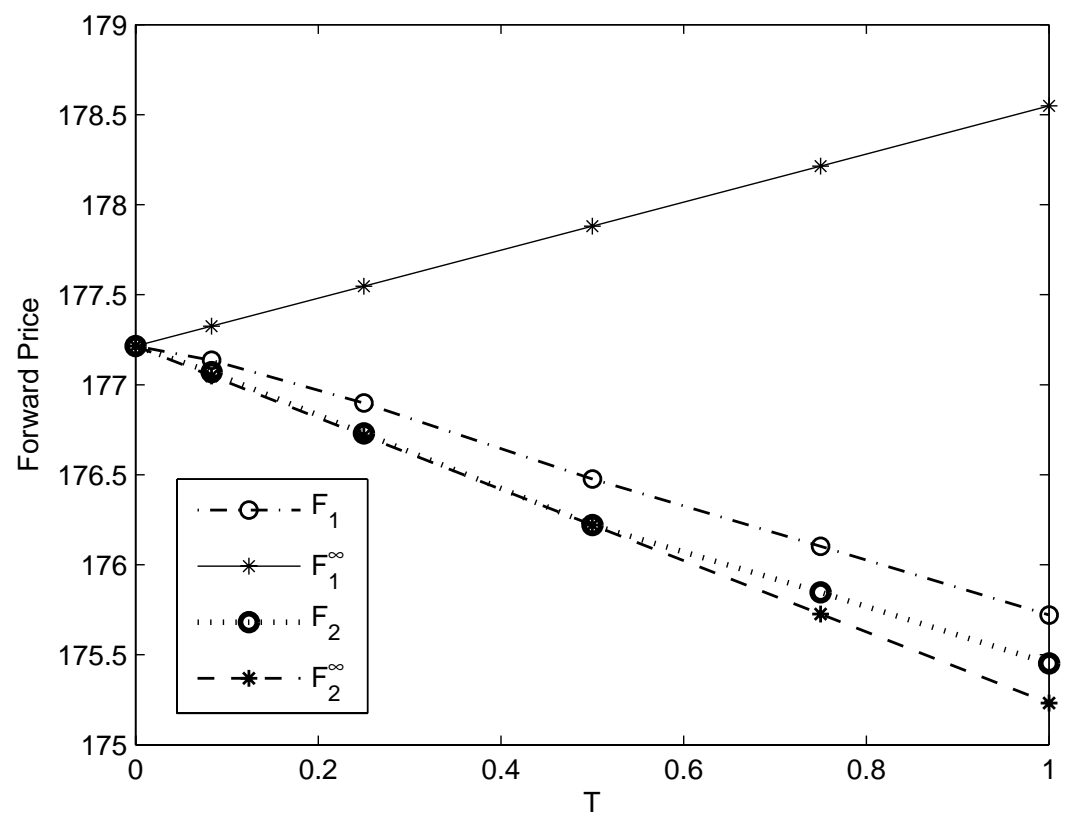

Figure 3: Forward prices, at $t=0$, plotted for time to maturities between $T=0$ and $T=1$ year. The initial value of the state variable is set to $Z_{0}=0.995 \times K_{12}$ in all curves and the current spot price is $S_{0}$. The current regime for the curves $F_{1}$ and $F_{1}^{\infty}$ is $k=1$, and the current regime for the curves $F_{2}$ and $F_{2}^{\infty}$ is $k=2$. Each point on the curves $F_{1}$ and $F_{2}$ is calculated from 600,000 paths of the underlying spot price process. Parameters are set according to parameter set I. 


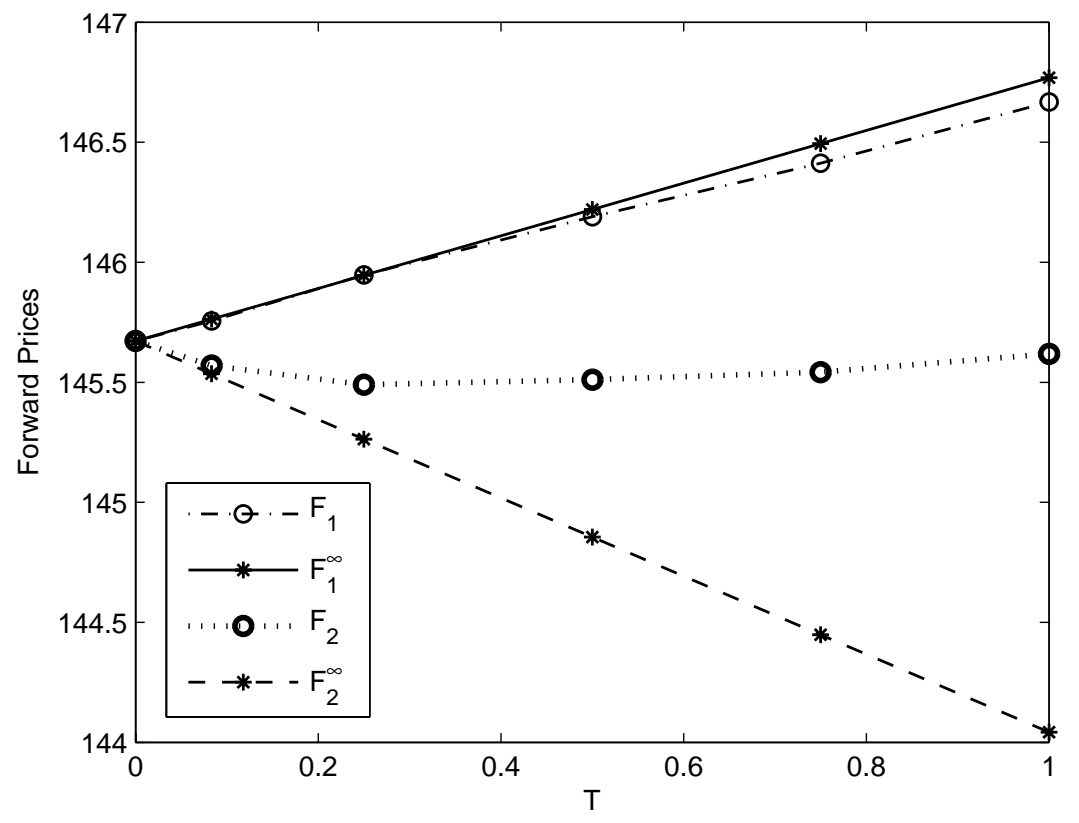

Figure 4: Forward prices, at $t=0$, plotted for time to maturities between $T=0$ and $T=1$ year. The initial value of the state variable is set to $Z_{0}=1.05 \times K_{21}$ in all curves and the current spot price is $S_{0}$. The current regime for the curves $F_{1}$ and $F_{1}^{\infty}$ is $k=1$, and the current regime for the curves $F_{2}$ and $F_{2}^{\infty}$ is $k=2$. Each point on the curves $F_{1}$ and $F_{2}$ is calculated from 600,000 paths of the underlying spot price process. Parameters are set according to parameter set I. 


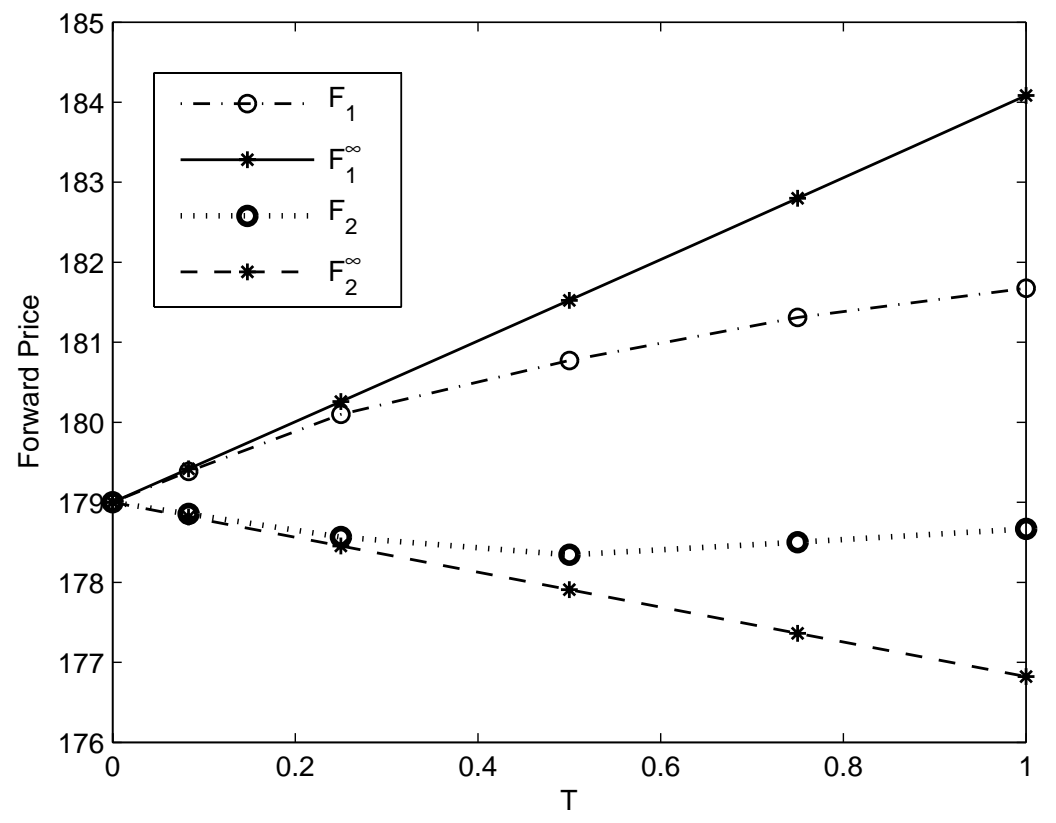

Figure 5: Forward prices, at $t=0$, plotted for time to maturities between $T=0$ and $T=1$ year. The initial value of the state variable is set to $Z_{0}=1.05 \times K_{21}$ in all curves and the current spot price is $S_{0}$. The current regime for the curves $F_{1}$ and $F_{1}^{\infty}$ is $k=1$, and the current regime for the curves $F_{2}$ and $F_{2}^{\infty}$ is $k=2$. Each point on the curves $F_{1}$ and $F_{2}$ is calculated from 600,000 paths of the underlying spot price process. Parameters are set according to parameter set II. 


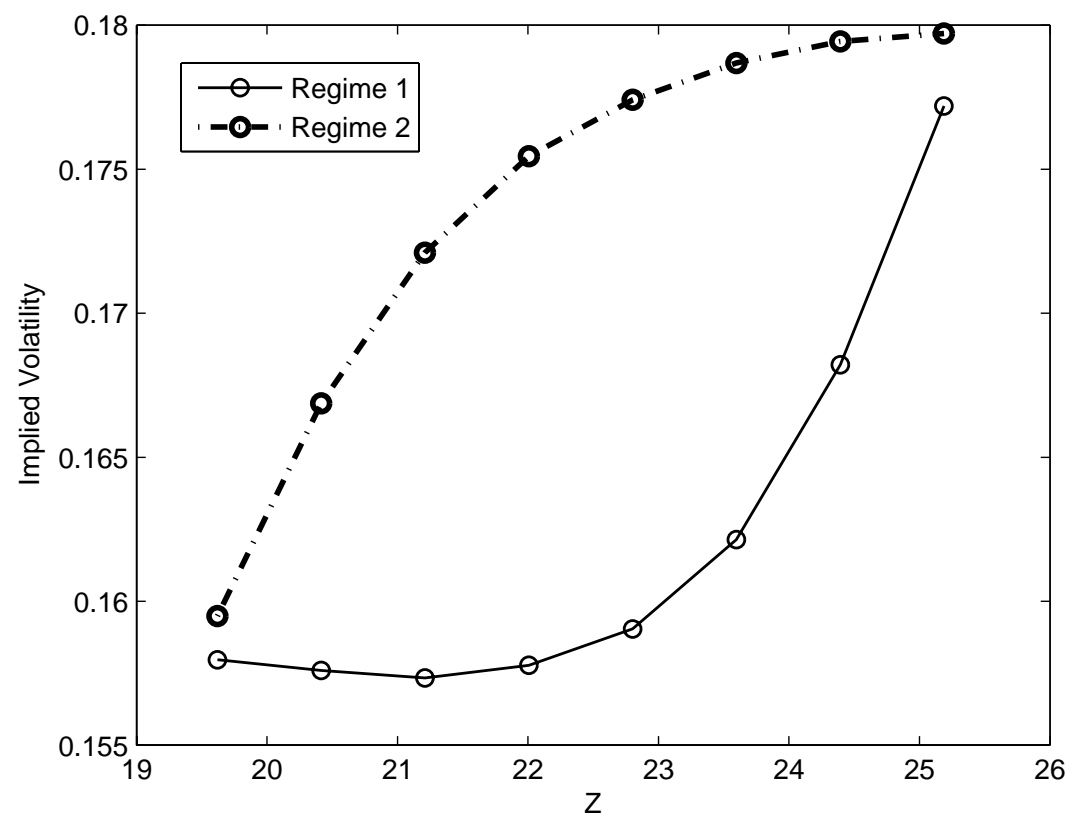

Figure 6: At-the-money implied volatilities as functions of $Z_{0}$ when the initial regime is either $k=1$ or $k=2$. Each implied volatility was obtained from simulated forward options and using Blacks formula. The initial forward price was also simulated. Each single price was calculated from Monte Carlo simulation using 600,000 paths. Parameters are set according to parameter set I. The options have a time to maturity of 6 months. 


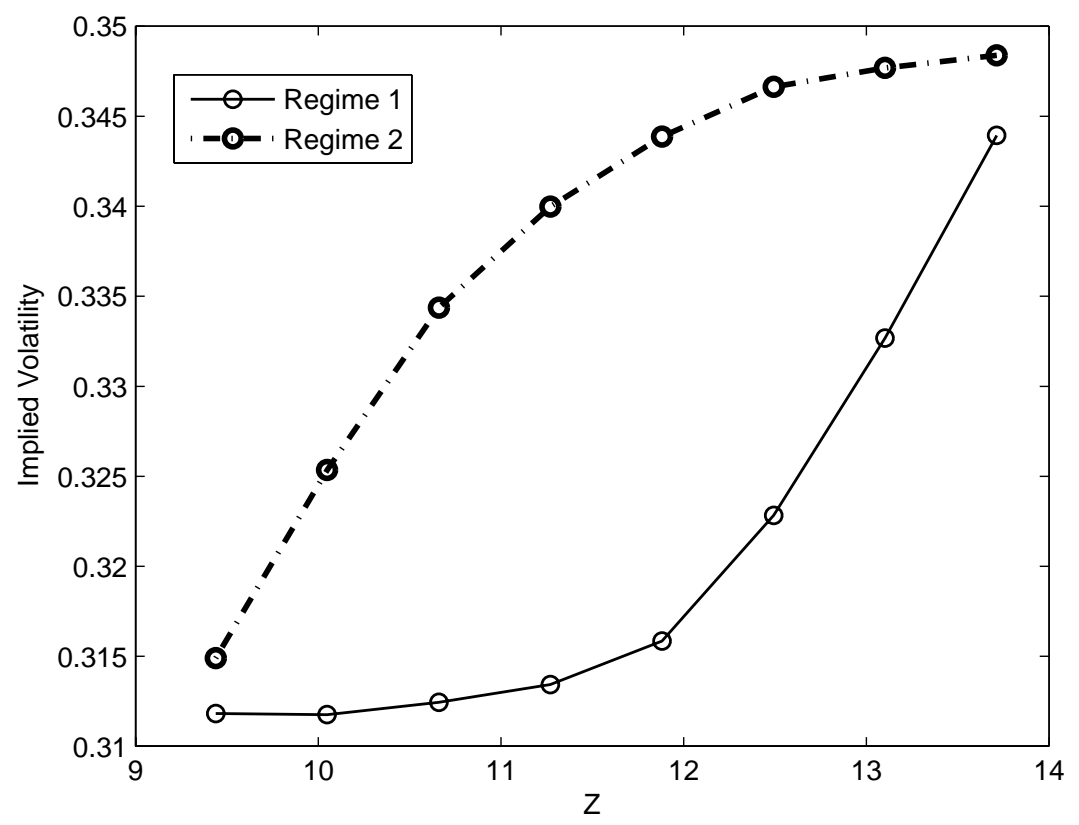

Figure 7: At-the-money implied volatilities as functions of $Z_{0}$ when the initial regime is either $k=1$ or $k=2$. Each implied volatility was obtained from simulated forward options and using Blacks formula. The initial forward price was also simulated. Each single price was calculated from Monte Carlo simulation using 600,000 paths. Parameters are set according to parameter set I. The options have a time to maturity of 6 months. 